\title{
Editorial
}

\section{EcoHealth and the Influenza A/H5N1 Dual Use Issue}

Highly pathogenic avian influenza, A/H5N1, more than any other infectious agent, has galvanized health authorities around the world to work together in a true One Health alliance. The recognition, soon after its discovery, of the involvement of wild birds, domestic poultry, and humans in its transmission and evolution strongly suggested that a systems approach is essential to fully understand the risk of a pandemic caused by the pathogen. The continued presence of the virus in wild birds and poultry farms, and its repeated spillover to people led to an influenza A/H5N1 pandemic being characterized by WHO director Margaret Chan as one of three global health crises looming on the horizon (McMichael et al. 2008).

The emergence of $\mathrm{H} 5 \mathrm{~N} 1$ influenza $\mathrm{A} / \mathrm{H} 5 \mathrm{~N} 1$ added enormous impetus to those who have been advocating for some time, an ecological or EcoHealth approach as the best way to tackle the increasing risk from a range of emerging infectious diseases. But as these new partnerships among ecologists, social scientists and laboratory scientists have begun to ripen, controversy has repeatedly emerged around the potential for the so-called dual use of research findings; i.e., their use to benefit health and the environment, or their use as agents of bioterrorism. In the last few weeks, it is influenza $\mathrm{A} / \mathrm{H} 5 \mathrm{~N} 1$ which is at the center of the controversy. What are the implications for EcoHealth researchers, and for the process of publishing our work?

The ability of a particular influenza virus strain to freely transmit between humans is central to the risk that this virus poses to humans. The highly pathogenic strain of influenza A/H5N1 causes around 80\% mortality in chickens and is highly transmissible within and among flocks. Its case fatality rate in humans may be as high as 60\%; however, the method used to establish this has been criticized as highly selective perhaps overstating the genuine human mortality rate by orders of magnitude (Palese and Wang
2012). Remarkably, little is known about the case fatality rate in the wider community of exposed people, including people who remain asymptomatic (Palese and Wang 2012). In any case, $\mathrm{H} 5 \mathrm{~N} 1$ has, to date, poor transmissibility between humans.

Some have suggested that improved understanding of human transmission will lead to mitigation strategies such as targeted vaccines or therapeutics. Identification of the genetic changes necessary for enhanced transmissibility also creates potentially important advances for surveillance or ecological control. Recent work has, therefore, focused on identifying the specific determinants of transmission of H5N1 using ferrets as models for humans in the US and the Netherlands (Osterholm and Henderson 2012). This work involved genetic manipulation of the pathogen to produce a virus capable of successful ferret to ferret transmission as a reasonable model of how this might occur in the humans. However, an expert committee of the US Academy of Sciences proposed the deliberate expansion of pathogen transmission to other species as one of seven classes of experiments that should automatically trigger extensive review and discussion before it can be undertaken or, if carried out, before it is published (Committee on Research Standards and Practices to Prevent the Destructive Application of Biotechnology 2004). This recent work on transmission studies in ferrets has, therefore, raised serious concerns over biosecurity and biosafety. Fears have been raised that virus constructs could be used deliberately by bioterrorists or accidentally escape from research laboratories. An initial response was a partial moratorium on animal transmission studies on $\mathrm{H} 5 \mathrm{~N} 1$ for 60 days.

For research to progress safely it is necessary to weigh the risks and benefits and if possible, manage and control them. There are three major perceived risks associated with the current work on $\mathrm{H} 5 \mathrm{~N} 1$ transmissibility. First, it has 
been postulated that complete publication of the genetic sequence of the ferret-transmittable virus could be used to reconstruct it for use in bioterrorism. Pressure on relevant journals has resulted in agreement to only partially publish the sequence data, in the hope that this would thwart such a possibility. However, there is no guarantee that ferrettransmittable H5N1 will have similar transmission among humans (Palese and Wang 2012), and the missing data may be available through other informal sources. On the other hand, some see this restriction as an infringement on free scientific speech, with an added risk that complete data will be confined to the military (Anon. 2012). Whatever the case, limited publication undoubtedly poses a real challenge for journal editors if it is to become an accepted process (Anon. 2012).

The second risk is associated with the deliberate removal of a modified strain from a research laboratory for use as a bioterrorism weapon. Bioterrorism is at least 2,000 years old, and was an important strategy in the Cold War (Committee on Research Standards and Practices to Prevent the Destructive Application of Biotechnology 2004). Following the deliberate release of anthrax spores in 2002, the United States developed strict regulations around potential bioterrorist agents, including H5N1 (http://www.selectagents.gov/ select $\% 20$ agents\%20and\%20Toxins\%20list.html). Other countries including the UK and Australia have similar lists and regulations. Perhaps the issue here is not the lack of a risk mitigation strategy but its effective application nationally and internationally.

The third risk involves the accidental escape of such a virus from a research laboratory, including through infection of a laboratory worker. While all laboratories working in this field have elements of biocontainment and biosafety, are they adequate and are they enforced? There have been several high profile pathogen escapes from laboratories in recent years, including the viruses that cause SARS and Foot and Mouth Disease.

There is a significant variation in biocontainment and biosafety regulations, both within and between countries. For example, there is not even agreement on whether the influenza H5N1 strain warrants Pathogen Containment level 3 or 4 (the threshold for at which full biocontainment suits must be worn). In many countries, regulations that pertain to medical laboratories differ from those in veterinary laboratories. Regulations that apply to genetically modified organisms differ or even conflict with those applying to pathogens exotic to the country, and both of the above are rarely harmonized with those regulations applying to "select agents," where they exist. Furthermore, there are often different regulatory authorities managing the plethora of rules, adding further confusion and misunderstanding.

But there is a case for undertaking such research. There is abundant evidence that the world faces an unprecedented and increasing risk from emerging infectious diseases, including repeated waves of influenza pandemics. It is also clear that the tools currently available for infectious disease mitigation and control (e.g., vaccines, antibiotics, rapid surveillance) are not adequate to prevent some pathogens from becoming pandemic (e.g., SARS), and causing substantial social disruption. History teaches us that research on potentially pandemic pathogens can enhance the available control strategies. For the EcoHealth researcher, this research includes a better understanding of disease ecology to help prevent spillover from animals to humans, and a refined understanding of social science underpinning disease transmission, to better define strategies that reduce social connectivity once spillover has occurred.

Examples abound of where containment laboratory approaches have been successfully fused with EcoHealth approaches leading to better understanding and mitigation of risk for emerging pathogens. For example, work on two emerging paramyxoviruses, Hendra and Nipah viruses, has involved experimental infections under BSL-4 conditions, ecological studies of the wildlife host reservoir, studies of the environmental and ecological drivers of emergence, and social science approaches to identify risk factors for infection. For Hendra virus, biocontainment laboratory research has identified the viral attachment protein, which has led to an effective vaccine for the livestock amplifier hosts which also has potential therapeutic value for human treatment. This success does not, of course, demonstrate that what we are doing in our research laboratories should or should not continue, poses an unacceptable risk, or that the research will prevent a catastrophic pandemic. Is it time to "take a break" and ponder the issue? Always, but we should do so with an eye on the benefits as well as the dangers, and with an understanding that whatever we change, it is likely to have impacts well beyond that of research on H5N1. At the same time, a moratorium on laboratory work, or publication of sequence data that might be deemed high risk for dual use does not preclude us from continuing work on the environmental determinants of disease spillover or spread. This might actually be a good opportunity to highlight the alternative management approaches focused on the humananimal-environment interface that EcoHealth champions. 
Whatever the final outcome of debates over dual use of molecular research on avian influenza, EcoHealth investigators working with zoonotic diseases should be knowledgeable about and engage in the policy discussions regarding influenza $\mathrm{A} / \mathrm{H} 5 \mathrm{~N} 1$ and other select agents. Our role in developing a deeper understanding of these pathogens, and novel control strategies for them is far from peripheral. Indeed, as we continue to identify new zoonotic pathogens carried by wildlife and to tease apart the ecological and social complexity underlying their emergence, our role in mitigating the risks to public health will surely become more central.

Martyn Jeggo

Australian Animal Health Laboratory

CSIRO Livestock Industries, PMB 24, Geelong, VIC 3220, Australia

Colin Butler

National Centre for Epidemiology and Population Health, College of Medicine Biology and Environment Australian National University, Canberra, Australia

Fang Jing

Institute for Health and Development Studies Kunming Medical University, Kunming, China
Phil Weinstein

University of South Australia, Adelaide 6009, Australia

Peter Daszak

EcoHealth Alliance, 460 West 34th Street, New York, NY, USA

e-mail: daszak@ecohealthalliance.org.

\section{REFERENCES}

Anonymous. (2012). Flu papers warrant full publication [editorial]. Nature 482:439-439.

Committee on Research Standards and Practices to Prevent the Destructive Application of Biotechnology (2004) Biotechnology Research in an Age of Terrorism, Washington, DC: National Academy of Sciences

McMichael AJ, Neira M, Heymann DL (2008) World Health Assembly 2008: climate change and health. The Lancet 371:18951896

Osterholm MT, Henderson DA (2012) Life sciences at a crossroads: respiratory transmissible H5N1. Science 335:801-802

Palese P, Wang TT. (2012). H5N1 influenza viruses: facts, not fear. Proceedings of the National Academy of Sciences. doi:10.1073/ pnas.1121297109.

Published online: May 11, 2012 